\section{Treatment options for females with hypogonadotropic hypogonadism and thromboembolism}

\author{
Sheila M. Quinn, ${ }^{1}$ Daniel J. Rubin² \\ ${ }^{1}$ Crozer-Keystone Health System, \\ 2Division of Endocrinology, \\ Temple University Hospital, \\ Philadelphia, USA
}

\section{Abstract}

Hormone therapy of the female Kallmann's Syndrome patient in the setting of a pulmonary embolism presents a challenge. A female with hypogonadotropic hypogonadism is usually presented with two treatment options - combined gonadal hormone replacement or pulsatile gonadotropin releasing hormone. When a patient incurs a contraindication to hormonal therapy, the management of their endocrine disorder becomes much more challenging. Providers and patients must consider the advantages and disadvantages to each treatment option, including the possibility of not treating at all. Given the lack of literature on this rare combination of events, herein we review Kallmann's Syndrome and its therapeutic options, as well as the hypercoagulability associated with hormone therapy to guide our decision-making process in a true case scenario.

\section{Case Report}

A 26-year-old Caucasian female with a past medical history of Kallmann's syndrome (KS) presented to our primary care clinic for followup after a recent hospital admission for pulmonary embolism. The patient was diagnosed with KS at age sixteen, and underwent hormonal induction of puberty with a subsequent maintenance regimen of oral contraceptives. At the time of admission she had been taking 35 mcg ethinyl estradiol and 180 mcg progestin for several years. She presented to the emergency department with shortness of breath and right-sided chest pain and was diagnosed with a pulmonary embolism. The patient was a nonsmoker with no personal or family history of hypercoagulable disorders or thromboembolic events. She denied any recent prolonged immobility or surgeries. A complete hematologic work-up was performed, yielding no abnormalities. The patient was started on warfarin for a planned six-month course, oral contraceptive pills were discontinued indefinitely, and she was discharged with instructions to follow-up with her primary care physician.

\section{Discussion}

$\mathrm{KS}$ is a rare disorder, with an incidence ranging from 1:10,000 to $1: 86,000$, and a male to female ratio of roughly 4:1.1 Characterized by hypogonadotropic hypogonadism and anosmia, KS patients are usually treated with hormone replacement regimens. The most common option in females not desiring immediate fertility is gonadal hormone replacement, as is done in their male counterparts. ${ }^{2}$ Females are started on unopposed estradiol, and doses are gradually increased until normal menses occur. When breast development is perceived as optimal, a progestin is added for endometrial protection. At this point, the progestin therapy can be taken in a continuous or cyclical fashion at the discretion of the patient and provider. ${ }^{3}$ The estrogen therapy however, should be continuous, as premature discontinuation can halt sexual maturity, initiate menopausal symptoms, and increase the risk of fractures in the developing female. ${ }^{4}$

The challenge presented in our case is that hormone therapy is associated with significant hypercoagulability. According to the World Health Organization Medical Eligibility Criteria (WHO MEC) for use of combination oral contraceptives (COC), patients with a personal history of deep vein thrombosis, or pulmonary embolism are assigned risk category 4 , a condition which represents an unacceptable health risk if the contraceptive method is used. 5

In order to adequately evaluate our patient's risk, we must evaluate existing treatment options for female hypogonadotropic hypogonadism, the most common being combined oral contraceptives (COC's). Many formulations of estrogen-progestin COC's are available today. Major characteristics that differentiate one formulation from another include the dosage of ethinyl estradiol (EE), the standard estrogen used in all COC's in the United States, and the generation of the progestin. The estrogen has changed significantly since COC's were first introduced in the United States in the mid 20th century, as current COC's contain 5-10 times less estrogen than original formulations. ${ }^{6}$ Although low-dose oral contraception has become common terminology, its origins are antiquated, as all of today's COC's in the US are manufactured with less than $50 \mathrm{mcg}$ of EE. ${ }^{7}$ Some formulations contain as little as $20 \mathrm{mcg}$ of EE, though their ability to optimally inhibit ovulation has been debated. ${ }^{8}$ Switching our patient to a lower EE-dose COC may offer a disadvantage in treating her KS and an advantage by decreasing her risk for future thrombotic events.
Correspondence: Sheila M. Quinn, CrozerKeystone Health System, 501 North Lansdowne Ave, Drexel Hill, Pennsylvania 19026 USA.

Tel. +1.607.342.2271.

E-mail: sheila.quinn@crozer.org

Key words: Kallmann's syndrome, female, thromboembolism, hormone therapy, oral contraceptive.

Conflict of interest: the authors report no conflicts of interest.

Received for publication: 30 September 2011. Revision received: 28 0ctober 2011.

Accepted for publication: 28 October 2011.

This work is licensed under a Creative Commons Attribution NonCommercial 3.0 License (CC BYNC 3.0).

(C) Copyright S.M. Quinn and D.J. Rubin, 2011 Licensee PAGEPress, Italy

Endocrinology Studies 2011; 1:e15

doi:10.4081/es.2011.e15

As discussed above, the role of estrogen therapy in the hypogonadotropic female is to maintain sexual maturity and maximize bone health. Data suggest the latter is optimized at a minimum dose of $30 \mathrm{mcg}$ EE in endocrinologically normal patients taking COC's for contraception. ${ }^{9}$ Cromer and colleagues demonstrated that normal adolescent and young adult women on a COC with $30 \mathrm{mcg} \mathrm{EE}$ and $1.5 \mathrm{mg}$ desogestrel showed no significant difference in percentage change of bone mineral density at one and two years of use, when compared to age-matched controls. ${ }^{9}$ These findings were supported by a similarly designed cohort study in slightly older subjects (18-31 years of age) also demonstrating that $30 \mathrm{mcg}$ EE appeared to be adequate for bone mineral density accrual. ${ }^{10}$ Furthermore, other studies found that young women using $20 \mathrm{mcg} \mathrm{EE}$ have less increase in bone mineral density than non-users, suggesting that $20 \mathrm{mcg}$ EE may inhibit normal bone development. ${ }^{11,12}$ Current knowledge about the effects of COC's on bone health must be extrapolated to women with KS because there are no published studies in KS patients.

Although lower-dose EE COC preparations may offer a disadvantage in terms of bone mineral density, they may be advantageous in regards to a reduction in hypercoagulability. Among healthy, non-pregnant women between 20-24 years old who do not use COC's, the incidence of thromboembolic events is about 0.3 per 10,000 per year. ${ }^{13}$ With the addition of a 30 mcg EE-containing $\mathrm{COC}$, their risk increases by 3-6 fold, with a general consensus that the risk is highest in the first year of use, and remains higher than non-users until discontinuation. ${ }^{13}$ Reducing the EE dose to $20 \mathrm{mcg}$ 
has had mostly positive effects regarding the propensity to clotting while on COC's. Norris and Bonner found that reducing the EE dose from $30 \mathrm{mcg}$ to $20 \mathrm{mcg}$ in a COC significantly lowered the levels of clotting factors $\mathrm{X}$ and VII in young healthy women. ${ }^{14}$ This supports the conclusions articulated by a 1993 prospective randomized trial also finding a significant decrease in hemostasis in 20 mcg EE pills compared to $30 \mathrm{mcg}$ EE. 15

In addition to estrogens, progestins have also been under scrutiny for their role in increasing thrombogenicity among female COC-users. Risk is projected to be even higher than the aforementioned 3-6 fold with COC formulations containing third generation progestins, as opposed to their second-generation predecessors. $^{16}$ In the previously mentioned study by Norris and Bonner, when the dose of EE was maintained at $30 \mathrm{mcg}$, and only the progestin component was manipulated, the women who took a COC containing third generation progestins (i.e. desogestrel) had significantly higher levels of clotting factors VII and $\mathrm{X}$ than those women taking a $\mathrm{COC}$ with a second generation progestin (i.e. gestodene). ${ }^{14}$ Notably, this contradicts one of the earliest studies on this topic, which argued that even though the two most influential coagulation inhibitors (Protein C and AT-III) could not adequately counter the increase in hemostasis induced by COC's, the biological levels did not differ between users of second versus third generation progestins. ${ }^{17}$ The attempt to definitively decipher the origins and limitations of the thrombogenicity associated with COC's has dominated much of the contraceptive literature in the past decade and may be an important contributor to our patient's therapy. In summary, decreasing the EE dosage and changing the progestin generation may significantly decrease our patient's chances of another thombotic event, but may also compromise critical bone development.

If we abandon the option of COC's altogether, we are left to evaluate the second widely accepted therapy for hypogonadotropic hypogonadism - pulsatile gonadotropic-releasing hormone (GnRH). GnRH has been used in both males and females. Usually, however, it is reserved for those who desire fertility, a physiologically difficult state for most KS individuals to obtain. ${ }^{4}$ It requires an intact pituitarygonadal axis and normal reproductive anatomy. Should we pursue this option with our patient, she is left with her body's own (albeit stimulated) production of gonadotropes and sex steroids and a potentially enhanced state of fertility. The former provides an optimal situation for bone mineral density accrual, while the latter may or may not be desired by the patient.

Although there are no data to suggest that pulsatile GnRH is directly associated with hypercoagulability, it is important to note that the incidence of venous thromboembolism in a normal healthy pregnant woman is $1 / 1000$ (with a 1-2\% case mortality), a markedly higher rate than that of healthy females using COC's. ${ }^{18}$ Furthermore, should a sexually active female patient elect the GnRH therapy and not desire fertility, we come full circle to the position of needing to provide an effective method of contraception to a young woman with a contraindication to exogenous estrogen therapy. The WHO MEC helps guide this decision again, concluding that the copper IUD bears the lowest risk for our patient; with a risk category of 1- a condition for which there is no restriction for the contraceptive method. ${ }^{5}$

The last option besides COC's or GnRH is foregoing hormonal treatment of the hypogonadotropic hypogonadism. This would certainly minimize our patient's chances of future thromboembolisms. However, as discussed above, this risks the premature arrest of sexual maturation, the initiation of menopausal symptoms, and suboptimal bone development and protection. The potential physical and psychological effects that accompany this therapeutic option warrant a thorough and wellinformed discussion between patient and provider.

\section{Conclusions}

In summary, we face a difficult situation: a young woman in need of hormonal therapy with a definitive contraindication to such. Our goals are four fold: i) to maintain her secondary sexual development; ii) to prevent the premature induction of menopause, iii) to optimize bone health, and iv) to avoid future thromboembolic events. That being said, our patient appears to have a few options. First, she could remain on the COC's with the consideration of switching to a formulation with a lower EE dosage and/or a second generation progestin. With this choice, we must recognize that although the literature is limited, it appears that bone mineral density could be compromised, meriting emphasis on prophylactic screening and lifestyle measures. Second, the patient could opt for pulsatile GnRH therapy, acknowledging the likelihood of enhanced fertility, and be offered the appropriate contraceptive counseling. Lastly, she could discontinue all hormonal therapy, with knowledge that with the marked decrease in thrombogenicity, comes potentially life-altering changes in sexual development and bone health. More research is needed to better evaluate treatment options for hypogonadal patients needing hormone therapy after a thrombotic event.

\section{References}

1. Seminara SB, Hayes FJ, Crowley WF. Gonadotropin-releasing hormone deficiency in the human (idiopathic hypogonadotropic hypogonadism and Kallmann's syndrome): pathophysiological and genetic considerations. Endocr $\operatorname{Rev} 1998 ; 19$ : 521-39.

2. Petak SM, Nankin HR, Spark RF et al. American Association of Clinical Endocrinologists Medical Guidelines for Clinical Practice for the Evaluation and Treatment of Hypogonadism in Adult Male Patietns - 2002 Update. Endocr Pract 2002;8:440-56.

3. Pallais J, Au M, Pitteloud N et al. Isolated Gonadotropin-Releasing Hormone (GnRH) Deficiency Overview. Gene Reviews 2007 May 23 [updated 2010 0ct 14].

4. Fechner A, Long S, McGovern P. A review of Kallmann Syndrome: Genetics, Pathophysiology, and Clinical Management. Obstet Gynecol Surv 2008;63:189-94.

5. National Center for Chronic Disease Prevention and Health Promotion Division of Reproductive Health. Medical Eligibility Criteria for Contraceptive Use: Fourth Edition. Available from: http://www.cdc. gov/mmwr/preview/mmwrhtml/rr59e0528a 1.htm

6. Petitti DB. Combination EstrogenProgestin Oral Contraceptives. N Engl J Med 2003;349:1443-50.

7. Speroff L, Glass R, Kase N. Clinical Gynecologic Endocrinology and Infertility. 6th edition. Lippincott Williams \& Wilkins. Philadelphia: 1999. pp. 867-945.

8. Gallo MF, Nanda K, Grimes DA, Schulz KF. $20 \mathrm{mcg}$ versus $>20 \mathrm{mcg}$ estrogen combined oral contraceptives for contraception. Cochrane Database Syst Rev 2005: CD003989.

9. Cromer B, Blair J, McArdle J et al. A prospective comparison of bone density in adolescent girls receiving depot medroxyprogesterone acetate (Depo-Provera), levonorgestrel (Norplant), or oral contraceptives. J Pediatr 1996;129:671-6.

10. Berenson A, Radecki C, Grady J et al. A prospective controlled study of the effects of hormonal contraception on bone mineral density. Obstet Gynecol 2001;98:576-82.

11. Polatti F, Fillippa N, Gallina D, Nappi R. Bone mass and long-term monophasic oral contraceptive treatment in young women. Contraception 1995;51:221-4.

12. Cromer B, Stager M, Bonny A et al. Depot medroxyprogesterone acetate, oral contraceptives and bone mineral density in a cohort of adolescent girls. J Adolesc Health 2004;35:434-41. 
13. World Health Organization. Cardiovascular disease and use of oral and injectable progestogen-only contraceptives and combined injectable contraceptives. Results of an international, multicenter, case-control study. World Health Organization Collaborative Study of Cardiovascular Disease and Steroid Hormone Contraception. Contraception 1998;57:315-24.

14. Norris LA, Bonnar J. The effect of oestrogen dose and progestogen type on haemo- static changes in women taking low dose oral contraceptives. Br J Obstet Gynaecol 1996;103:261-7.

15. Basdevant A, Conard J, Pelissier C et al. Hemostatic and metabolic effects of lowering the ethinyl-estradiol dose from $30 \mathrm{mcg}$ to $20 \mathrm{mcg}$ in oral contraceptives containing desogestrel. Contraception 1993;48: 193-204.

16. Spitzer W, Lewis M, Heinemann L, Thorogood M. Third Generation oral con- traceptives and risk of venous thromboembolic disorder: an international case-control study. BMJ 1996;312:83-8.

17. Cohen H, Mackie IJ, Walshe K et al. A comparison of the effects of two triphasic oral contraceptives on haemostasis. $\mathrm{Br} \mathrm{J}$ Haematol 1988;69:259-63.

18. Walker ID. Venous and arterial thrombosis during pregnancy: epidemiology. Semin Vasc Med 2003;3:25-32. 